\title{
Composite nanostructure of vertically aligned carbon nanotube array and planar graphite layer obtained by the injection CVD method
}

\author{
V.A. Labunov ${ }^{1}$, B.G. Shulitski ${ }^{1}$, A.L. Prudnikava ${ }^{1}$, Y.P. Shaman ${ }^{1}$, A.S. Basaev ${ }^{2}$ \\ ${ }^{I}$ The Belarusian State University of Informatics and Radioelectronics, \\ Laboratory of Integrated Micro- and Nanosystems, \\ Minsk, 220013 Belarus, Phone/Fax: +375 17 202-10-05 \\ ${ }^{2}$ Technological Center, Moscow State Institute of Electronic Technology, \\ Moscow, K-498, 103498 Russia, Phone: +7(095) 532-8906; fax: +7(095) 913-2192
}

\begin{abstract}
The carbon nanostructure composed of an array of vertically aligned carbon nanotubes (CNTs) and a planar graphite layer (PGL) located at the top of the array has been obtained by the injection chemical vapor deposition method, realized using high temperature catalytic pyrolysis of xylene-ferrocene mixture. The carbon nature of the planar layer was identified using Auger electron spectroscopy. Scanning electron microscopy analysis enabled to ascertain peculiarities of CNT-PGL nanostructure morphology, in particular, the internal layer-built structure of PGL and its links with the underlying CNT array. The mechanism of CNT-PGL nanostructure formation was considered.
\end{abstract}

Keywords: carbon nanotubes, graphite, graphene, chemical vapor deposition, ferrocene, xylene.

Manuscript received 14.01.10; accepted for publication 25.03.10; published online 30.04.10.

The unique electrophysical, chemical and mechanical properties of carbon nanotubes (CNTs) predetermine a variety of the areas of their application [1]. These areas can be considerably extended due to composite nanostructures and branch architecture creation on the CNT basis, especially in a combination with different allotropic forms of carbon.

Presently, some of these composite nanostructures have been created, for example, CNTs filled with fullerenes, the so-called "peapods" [2], and nanostructures composed of an array of vertically aligned CNTs and a planar graphite layer located at the top of the array [3]. The composite nanostructure obtained by Kondo et al. [3] was grown using high temperature catalytic pyrolysis of acetylene at the low pressure $(1000 \mathrm{~Pa})$ by using localized bimetallic catalyst $\mathrm{Co} / \mathrm{TiN}$ preliminary deposited on $\mathrm{Si} / \mathrm{SiO}_{2}$ substrate.

This investigation is aimed at creation of the composite nanostructure like to that in ref. 3, but using the injection CVD method as the most simple and controllable one.

The composite carbon nanostructure was synthesized by the injection CVD method (with the injected catalyst) using a feeding solution of ferrocene
$\left(\left[\mathrm{Fe}\left(\mathrm{C}_{5} \mathrm{H}_{5}\right)_{2}\right]\right.$, Aldrich) in fluid hydrocarbon ( $p$-xylene $\left[\mathrm{C}_{8} \mathrm{H}_{10}\right]$, Aldrich) which was injected into the reaction zone of the tubular silica reactor of $10 \mathrm{~mm}$ in diameter. Argon was used as a gas-carrier with the constant flow $\left(100 \mathrm{~cm}^{3} \cdot \mathrm{min}^{-1}\right)$ through the reactor. The synthesis process was carried out at the temperature $850{ }^{\circ} \mathrm{C}$ for $60 \mathrm{~min}$ under atmospheric pressure by using $1.0 \mathrm{wt} . \%$ feeding solution. Wafers of $n$-type silicon covered with 100 -nm thermally grown $\mathrm{SiO}_{2}$ were used as substrates.

Under the mentioned above conditions, the composite nanostructure consisting of the array of vertically aligned CNTs and a self-organized planar layer located at the top of the array was obtained (Fig. 1).

The SEM image of the obtained composite nanostructure (Fig. 1a) is presented in a convenient aspect angle for its analysis. The cleavage of nanostructure was done in a way that permits to observe not only the planar layer and CNT array but the substrate surface open after deleting a part of the composite nanostructure.

From Fig. 1a, one can conclude that the composite nanostructure has a height of $\sim 2.3 \mu \mathrm{m}$, it is very firm (not destroyed during the mechanical cleavage), and 
CNT array has good adhesion both to the upper planar layer and to the substrate. Using the Auger electron spectroscopy (AES) method (Fig. 1b), it was established that the only element of the planar layer is carbon with the negligible amount of Fe. It means that we obtained CNT-planar graphite layer (PGL) nanostructure, which we designate as "CNT-PGL nanostructure".

In Fig. 2, the SEM images of CNT-PGL nanostructure for various aspect angles and magnifications are presented. In Fig. $2 \mathrm{a}$, it is seen that PGL represents the layer-built structure, which was designated in ref. 3 as a "graphene multi-layer". One should note that the surface of this layer is very rough in a nanoscale. The thickness of PGL is close to $\sim 25 \mathrm{~nm}$. Fig. $2 b$ demonstrates the incorporation of separate CNTs into PGL through the catalyst nanoclusters, which ensures strong adhesion of CNTs to PGL. Data of Fig. 2 give important information to understand the mechanism of CNT-PGL nanostructure formation.

A simple scheme of the proposed mechanism is presented in Fig. 3. This mechanism is based on the analysis of CNT growth mechanisms. Despite some peculiarities, in general the mechanisms of CNT growth are considered as the multistage ones [4-8].

In the first stage, after the feeding solution of ( $p$ xylene in the mixture with ferrocene) is injected into the reaction zone, the thermal homogeneous gas-phase decomposition of ferrocene under the temperature of around $500^{\circ} \mathrm{C}$ is going on. As a result, $\mathrm{Fe}$ is deposited onto $\mathrm{Si} / \mathrm{SiO}_{2}$ substrate surface creating a layer of $\mathrm{Fe}$ catalyst nanoclusters (Fig. 3a(1)).
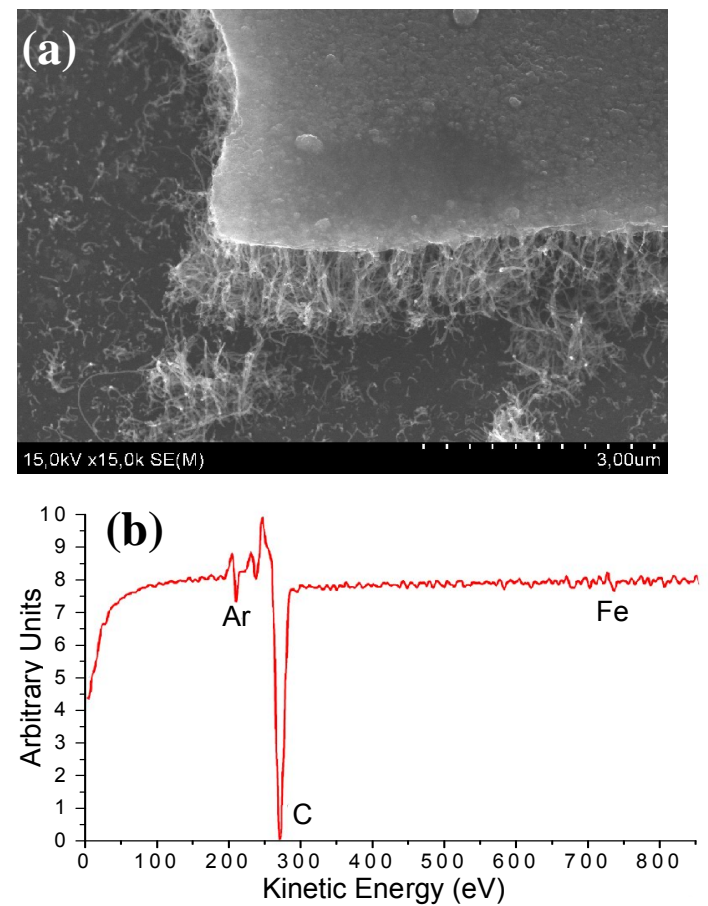

Fig. 1. Composite nanostructure consisting of an array of vertically aligned CNTs and a planar layer on top: (a) SEM image, (b) Auger spectrum of the planar layer surface.
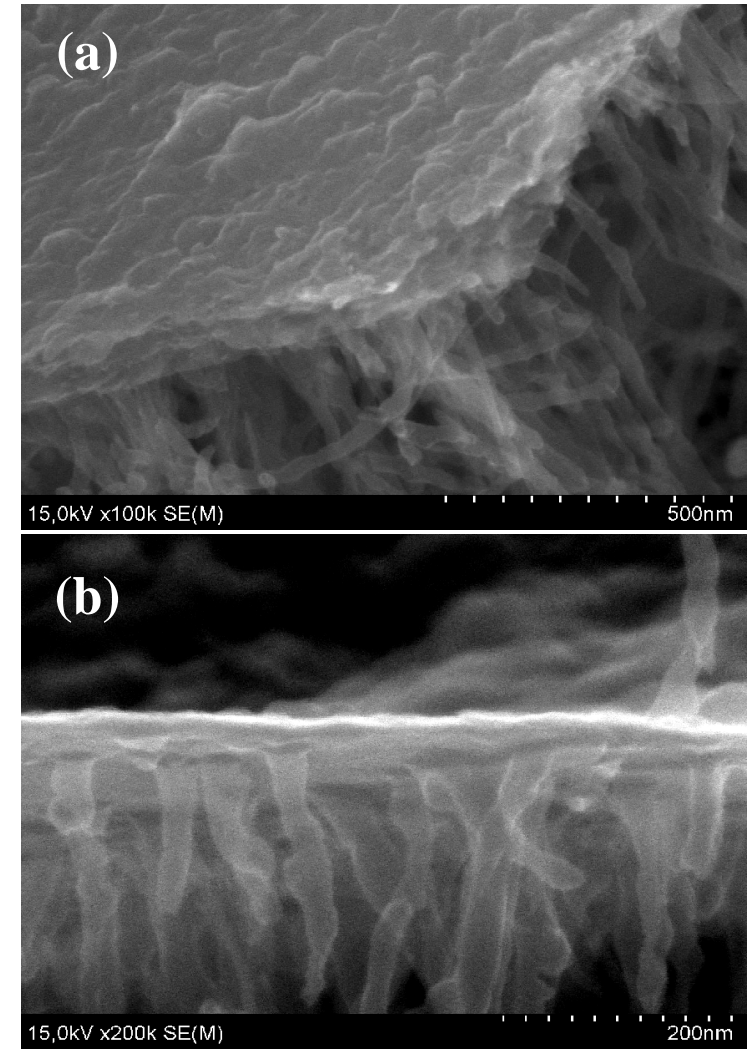

Fig. 2. CNT-PGL nanostructure at different aspect angles and magnifications (SEM): (a) top view of the planar graphite layer; (b) view of CNT-PGL junction.

In the second stage, Fe nanoclusters decompose carbon source (hydrocarbons), creating carbon atoms and dissolve them up to a certain limit. Dissolved carbon atoms activate $\mathrm{Fe}$ in nanoclusters and under the temperature close to $800{ }^{\circ} \mathrm{C}$ form fluid-like Fe carbide (Fig. 3a(2)). Let's designate this process as "carbidization cycle".

In the third stage, due to the metastable character of fluid-like Fe carbide, the process reversed to the carbide formation leads to the carbide decomposition by segregating a part of the dissolved carbon (Fig. 3a(3)). The segregated carbon atoms diffuse to the catalyst surface and extrusion of one of the carbon allotropic forms of different dimensionalities from the catalyst surface starts ("carbonization cycle"). This stage is designated as the nucleation one. CNT growth in the nucleation stage has various interpretations by different investigators. According to refs. 4 and 5, carbon extrusion leads to the fullerene hemisphere formation, which nucleates the CNT growth. This mechanism is based on the in-situ TEM observations [5].

The main idea of ref. 7 is that this extrusion of carbon leads to the direct growth of CNTs on the catalyst surface. The gas-phase precursor, $\mathrm{C}_{\mathrm{gas}}$, is deposited on the open sides of the catalyst surface being converted to an activated surface-bound form, $\mathrm{C}^{*}$, which dissolves into the Fe carbide nanocluster maintaining it in a fluid- 
(a)
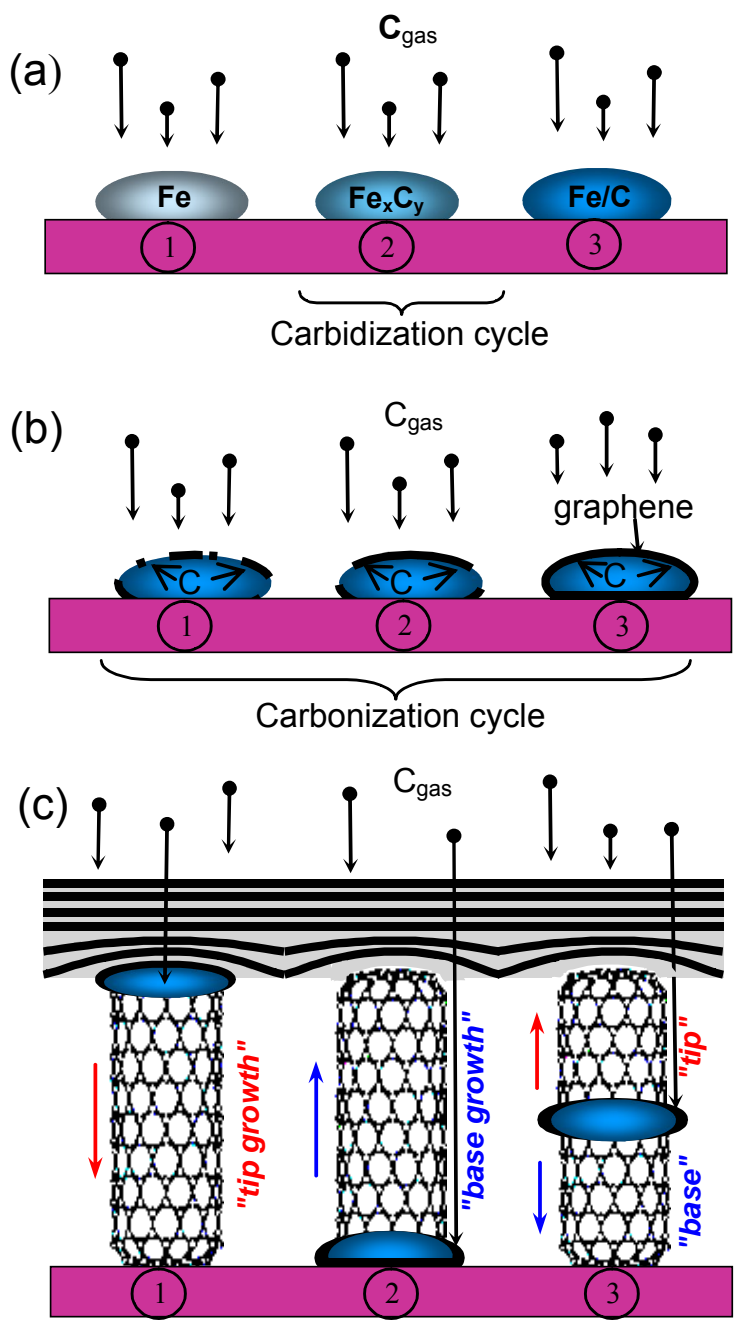

Fig. 3. Scheme of the CNT-PGL nanostructure formation: (a) Fe catalyst nanoclusters formation on the substrate surface, (b) graphene layer formation on the surface of $\mathrm{Fe}$ catalyst nanoclusters, (c) PGL and CNT array growth with lifted up PGL by the growing CNT array.

like phase with the oversaturated concentration of carbon. Under the concentration gradient, $\mathrm{C}^{*}$ diffuses through the catalyst nanoclusters and directly incorporates into the growing CNT. In ref. 7, a very important aspect of CNT growth is considered. It is shown that $\mathrm{C}^{*}$ can form on the catalyst nanocluster surface the amorphous graphite patches which can grow up and fully cover surface, what will lead to deactivation of the catalyst and termination of CNT growth. In a general case, it will lead to termination of any carbon allotropic form growth. Let's designate this process as "graphitization cycle" of CNT growth.

In ref. 8, it is undoubtedly declared that the islands of graphene layer are formed on the catalyst surface as a result of carbon extrusion (Fig. 3b(1)). This gives rise to a carbon-concentration gradient in the catalyst allowing more carbon source to be decomposed and segregated.
In such a way, the area of the graphene layer is growing up on the catalyst surface (Fig. $3 b(2)$ ). This process will continue until all surface of the carbide nanocluster is covered with two-dimensional graphene nuclii (Fig. 3b(3)). We consider the latter approach as the most realistic one, because graphene, as a product of carbon extrusion, is the most energetically favorable carbon allotropic form to be created [9].

A stable graphene layer on the catalyst surface can be obtained, if it has perfect structure, i.e. contains only hexagonal ones (honeycomb lattice). The combination in the graphene layer of the hexagonal cells with the "imperfect" ones (penta-, hepta-, dodecagonal cells) makes it unstable, and it becomes a basic building block for graphitic materials of all other dimensionalities. It can be wrapped up into 0D fullerenes (buckyballs), rolled into 1D nanotubes or stacked into 3D graphite or soot [10].

The existence of the carbonization or graphitization cycles can be determined by several reasons. In particular, when the rate of carbon supply to the catalyst surface exceeds the rate of its diffusion (outflow) through the catalyst, more probably graphitization of the catalyst surface occurs. In the case of too slow rate of carbon supply to the catalyst surface, carbonization of catalyst surface would preferably occur, because the carbon extrusion products have enough time to form the most energetically favorable carbon allotropic form graphene.

Both rates depend on the numerous factors, such as the rate of hydrocarbon decomposition and partial pressure of its products, which in turn can be regulated by the temperature in the reaction zone, the rate of feeding solution injection, etc. Thus, regulating these parameters one may create conditions for carbonization or graphitization of the catalyst nanoclusters.

We use the considered mechanism of CNT growth as a basis for the developing the mechanism of CNTPGL nanostructure formation.

In ref. 3, the probable mechanism of CNT-planar "graphene multi-layer" nanostructure formation was briefly considered. The main feature of this mechanism is that after the planar layer formation (it is not considered in what a way) the CNT array is synthesized simply by the tip-growth mechanism [6].

Our vision of the mechanism of CNT-PGL nanostructure formation is different. Since the feeding solution is continuously supplied into the reaction zone during the CNT-PGL nanostructure formation, $\mathrm{C}_{\mathrm{gas}}$ and $\mathrm{Fe}$ atoms are continuously generated and take part in the growth process. In our opinion, the processes of PGL and CNT arrays formation are going on simultaneously. Under the realized conditions of CNT-PGL nanostructure formation, the graphene layer grown at the nucleation stage on the catalyst surface is transformed by some reasons into the planar graphite layer ("graphitization cycle") at the upper surface of catalyst and into CNTs at the interface between catalyst and substrate surface. 
While in the case of CNT growth the graphitization process leads to the catalyst deactivation and CNT growth termination, in the case of CNT-PGL nanostructure formation the graphitization process leads to the PGL creation.

PGL grows in the following way (Fig. 3c). In the course of CVD process realization, the carbon atoms from the gas phase precipitate on the substrate, successively creating by the epitaxial growth the layers of the "graphene multi-layer" nanostructure (PGL), which covers the substrate surface with the catalyst nanoclusters on it. In this case, the PGL thickness grows up during the synthesis process. Fe atoms also precipitate onto the surfaces of the growing graphene layers creating $\mathrm{Fe}$ nanoclusters, which are encapsulated between these layers (not shown in Fig. 3). This assumption is confirmed by Fig. 2a where the PGL surface is presented, which is very rough in a nanoscale. Evidently, the graphene layers of PGL replicate the underlying Fe nanoclusters. AES in Fig. $1 \mathrm{~b}$ confirms the existence of small amount of $\mathrm{Fe}$ in the CNT-PGL nanostructure.

All the time during PGL growth, carbon atoms from the gas phase diffuse through growing PGL to the catalyst facilitating CNT growth (Fig. 3c).

Iron atoms can diffuse through graphite layers only at temperatures above $1600{ }^{\circ} \mathrm{C}[10]$, what is much higher than our CVD condition $\left(850^{\circ} \mathrm{C}\right)$, therefore participation of iron from the gas phase in CNT growth is excluded.

There are some options of CNT growth under the CNT-PGL nanostructure formation. If the adhesion of the catalyst to PGL is stronger than to the substrate, the CNT growth starts at the catalyst-substrate interface towards the substrate (Fig. 3c(1)). The growing CNTs detach and lift up the catalyst nanoclusters and PGL from the substrate surface. As a result, the catalyst moves upwards in the direction opposite to the direction of CNT growth, what represents classical tip-growth mechanism [6]. If the adhesion of catalyst to the substrate is stronger than to PGL, the CNT growth starts at the catalyst-PGL interface towards PGL. The growing CNTs lift up PGL and detach catalyst from PGL. Catalyst remains at the substrate during the CNT growth (Fig. 3c(2)), what represents the classical base-growth mechanism [6]. There might be an intermediate situation as additional one to the case of Fig. 3c(1) when CNT growth happens at the both sides of catalyst starting at catalyst-substrate interfaces and continues on the catalyst-PGL interfaces. In this case, the CNT growth goes on by combination of the base- and tipgrowth mechanisms (Fig. 3c(3)), and catalyst might happen to locate somewhere in the CNT array between PGL and substrate.

Fig. 4 confirms the proposed mechanism of CNT growth under the CNT-PGL nanostructure formation. There is easily recognized situation that CNTs grow both from PGL and from substrate, and it looks like they are meeting each other just in the middle of the distance between PGL and substrate. It is important to note that the density of the CNT array growing from the substrate looks higher than source growing from PGL.

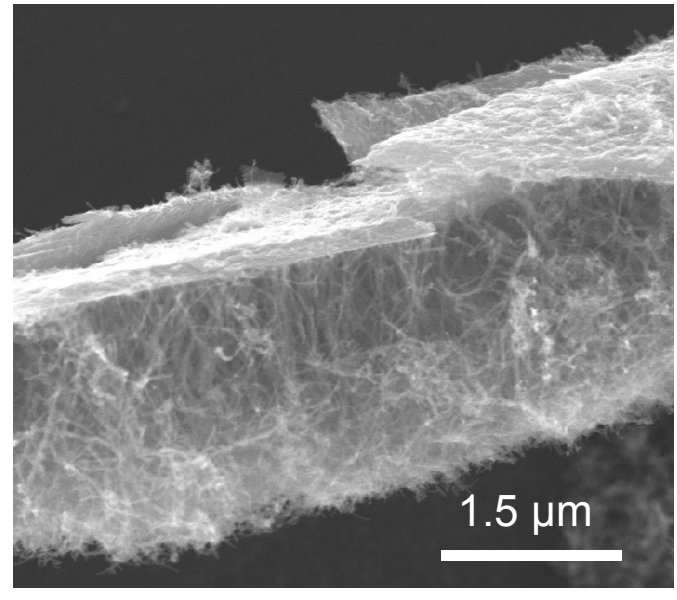

Fig. 4. View of the CNT array located between PGL and substrate.

Additional evidence that CNTs grow from PGL can be taken from Fig. 2b. On the contrary, Fig. 1a demonstrates that CNTs grow from substrate, because on the open side of the substrate surface one can observe the rests of the broken-down during the cleavage process CNTs that are very likely located at the catalyst nanoclusters.

The process of CNT growth continues until it terminates by some reasons. The growth cessation may be caused either by graphitization of the catalyst nanocluster surface or by limitations imposed by diffusion of carbon atoms to the catalyst clusters through PGL. At the beginning of the CNT-PGL nanostructure formation, when PGL thickness is small and the diffusion length of carbon atoms is larger than the PGL thickness, graphitization might be responsible for the cessation. As the PGL thickness is growing, the gasdiffusion controlled mode can prevail at the certain crucial thickness, and growth cessation would be caused by the limitations imposed by diffusion of carbon atoms through PGL. The proposed mechanism of the CNTPGL nanostructure formation is different from the mechanism proposed in ref. 3 for the same type of composite nanostructure. Our mechanism describing the growth of the planar "graphene multi-layer" combines both the base- and tip-growth mechanisms in different manifestations. We propose to designate this mechanism as the "combined CNT-PGL growth" mechanism.

In summary, for the first time the carbon nanostructures composed of an array of vertically aligned CNTs and a planar graphite layer (PGL) located at the top of the array (CNT-PGL nanostructures) have been synthesized by the injection CVD method. The mechanism of CNT-PGL nanostructures formation by injection CVD method is proposed, which is based on the analysis of all the stages of CNT growth mechanisms. From different interpretations of CNT growth mechanism, the most appropriate ones was chosen, according to which CNT growth goes on 
through the stages of metal carbide formation (carbidization cycle) and its decomposition with the following graphene layer creation at the catalyst surface (carbonization cycle). CNT-PGL nanostructure formation goes on by the transformation of the graphene layer into PGL at the upper surface of catalyst and into CNTs at the interface between catalyst and substrate surface. The proposed mechanism is designated as "combined CNT-PGL growth" mechanism. The obtained CNT-PGL nanostructure is expected to have unique electro-physical properties and therefore is likely to find many applications in electronics.

\section{Acknowledgements}

The authors acknowledge the BelMicroSystems R\&D Center, Minsk for giving us an opportunity to perform SEM investigations, and the Institute of Heat and Mass Transfer, Minsk, in particular Prof. S.A. Filatov, for EDX measurements.

\section{References}

1. S. Iijima, Helical microtubules of graphitic carbon // Nature 354, p. 56-58 (1991).

2. B. Smith, M. Monthioux, and D. Luzzi, Encapsulated $\mathrm{C}_{60}$ in carbon nanotubes // Nature 396, p. 323-324 (1998).

3. D. Kondo, S. Sato, Y. Awano, Self-organization of novel carbon composite structure: Graphene multilayers combined perpendicularly with aligned carbon nanotubes // Appl. Phys. Express 1, 074003 (2008).
4. O. Jost, A.A. Gorbunov, J. Möller, W. Pompe, X. Liu, P. Georgi, L. Dunsch, M.S. Golden, and J. Fink, Rate-limiting processes in the formation of single-wall carbon nanotubes: Pointing the way to the nanotube formation mechanism // J. Phys. Chem. B. 106, p. 2875 (2002).

5. A. Yasuda, N. Kawase, and W. Mizutani, Carbonnanotube formation mechanism based on in situ TEM observations // J. Phys. Chem. B. 106, 13294 (2002).

6. P.V. Fursikov, B.P. Tarasov // Intern. Sci. Journal for Alternative Energy and Ecology 10, p. 24 (2004), in Russian.

7. M. Stadermann, S.P. Sherlock, J.-B. In, F. Fornasiero, H.G. Park, A.B. Artyukhin, Y. Wang, J.J. De Yoreo, C.P. Grigoropoulos, O. Bakajin, A.A. Chernov, and A. Noy, Mechanism and kinetics of growth termination in controlled chemical vapor deposition growth of multiwall carbon nanotube arrays // Nano Lett. 9, p. 738-744 (2009).

8. S. Esconjauregui, C.M. Whelan, and K. Maex, The reasons why metals catalyze the nucleation and growth of carbon nanotubes and other carbon nanomorphologies // Carbon 47, p. 659-669 (2009).

9. A.K. Geim and K.S. Novoselov, The rise of graphene // Nature Materials 6, p. 183-191 (2007).

10. R. Andrews, D. Jacques, D. Qian, and E.C. Dickey, Purification and structural annealing of multiwalled carbon nanotubes at graphitization temperatures // Carbon 39, p. 1681-1687 (2001). 